\title{
Gridded Monthly Rainfall Estimates Derived from Historical Atoll Observations
}

\author{
WERNER E. COOK AND J. SCOTT GREENE \\ Department of Geography and Environmental Sustainability, University of Oklahoma, Norman, Oklahoma
}

(Manuscript received 7 August 2018, in final form 28 January 2019)

\begin{abstract}
To provide an analysis tool for areal rainfall estimates, $1^{\circ}$ gridded monthly sea level rainfall estimates have been derived from historical atoll rainfall observations contained in the Pacific Rainfall (PACRAIN) database. The PACRAIN database is a searchable repository of in situ rainfall observations initiated and maintained by the University of Oklahoma and supported by a research grant from the National Oceanic and Atmospheric Administration (NOAA)/Climate Program Office/Ocean Observing and Monitoring. The gridding algorithm employs ordinary kriging, a standard geostatistical technique, and selects for nonnegative estimates and for local estimation neighborhoods yielding minimum kriging variance. This methodology facilitates the selection of fixed-size neighborhoods from available stations beyond simply choosing the closest stations, as it accounts for dependence between estimator stations. The number of stations used for estimation is based on bias and standard error exhibited under cross estimation. A cross validation is conducted, comparing estimated and observed rains, as well as theoretical and observed standard errors for the ordinary kriging estimator. The conditional bias of the kriging estimator and the predictive value of kriging standard errors, with respect to observed standard errors, are discussed. Plots of the gridded rainfall estimates are given for sample El Niño and La Niña cases and standardized differences between the estimates produced here and the merged monthly rainfall estimates published by the Global Precipitation Climatology Project (GPCP) are shown and discussed.
\end{abstract}

\section{Introduction}

The purpose of this research is to produce monthly gridded rainfall estimates for the tropical Pacific Ocean with validated standard error estimates, derived solely from in situ atoll rainfall measurements. In selecting data from which to derive estimates, we have placed a higher priority on internal consistency of that data and consistency, in terms of expected rainfall climatology, between the observing stations and targeted estimate locations, than on maximal coverage of estimates having theoretical standard errors below some arbitrary threshold. In selecting the estimation procedure, we have opted for ordinary kriging, a technique first described by Matheron (1963), which has the advantage of producing a theoretical standard error value, kriging variance, based upon the variability of the data as a function of distance (the variogram) as well as the geometric distribution of data points used for estimation. The suitability of kriging variance as a predictor of actual standard error can be determined directly.

Corresponding author: Werner E. Cook, ecook@ou.edu
Claims of relative performance are not made, since similar products derived from in situ data do not appear in the literature at this time. Improvements in accuracy are constrained by the degree to which the data capture variations in rainfall at progressively smaller spatial scales, regardless of the estimation method used. Also, techniques that involve more complicated models (e.g., universal kriging) require more data to achieve the same level of optimization and are not necessarily as robust in their applicability as ordinary kriging. Space-time kriging (see Cressie and Wikle 2011) may offer an avenue to improving estimates by incorporating rainfall data from a temporal neighborhood around a particular month.

The sparseness of observing stations over tropical oceans, in concert with the multitude of spatial scales over which rainfall varies, limits the performance, in terms of accuracy, of the gridded products introduced here. Such estimates are subject to standard errors that sometimes exceed the magnitudes of the estimates themselves. Nevertheless, rainfall estimates based on in situ measurements are indispensable for determining confidence bounds of precipitation values obtained from numerical model or remote sensing algorithm outputs 
and for climatological comparisons. Confidence in standard error estimates (not the size of standard errors themselves) and a lack of bias are primary features we aim to achieve here; performance, in terms of suitability for a particular purpose, is determined properly by the end user.

Having more comprehensive coverage than other remote sensing datasets (radar, passive microwave, etc.), as well as resolution in space and time roughly fitting the requirements mentioned above, infrared satellite data contain information that could be helpful in indirectly inferring the scales and intensities of rainfall features, but they are problematic for two reasons. First, they do not exist for much of the history for which we wish to produce gridded values. Second, and more importantly, their usefulness for discriminating between contributions of different scales is limited by the smallest scales over which their joint statistics with in situ rainfall measurements are known. Ultimately, such joint statistics must be obtained by comparing the remote sensing datasets to in situ data subject to appropriate areal averaging (rainfall estimates derived from remote sensing data are areal estimates, not point estimates) and temporal aggregation. In turn, areal averages of in situ data and their error characteristics must be rooted in the comparison of point interpolations directly to point (rain gauge) measurements. In other words, the first step in the process of determining how the physical characteristics of the rainfall data affect interpolated estimates is to generate and validate point estimates using in situ data.

There are plentiful examples in the literature, such as Ly et al. (2011), Kastelec and Kosmelj (2002), Tang (2002), and Goovaerts (2000), of rainfall estimation using various forms of kriging, yet these papers use observations that are relatively dense in space compared to the data used in this paper. In contrast, analogous work to produce rainfall estimates over open oceans using only sparse rain gauge observations are lacking, with Morrissey (1991) and Morrissey et al. (1995) being notable exceptions; these focus on the estimation of uncertainty of spatial averages constructed from point measurements, where averaging domains contain many data points. While the lack of directly comparable research may indicate a presumption that monthly point rainfall estimates derived from rain gauge data gathered exclusively at atoll locations will severely limit the domains over which standard errors are sufficiently small to ensure suitability for a particular use, that situation does not diminish the importance of such estimates. These point estimates and associated standard errors provide directly verifiable bounds on the uncertainty of areal estimates derived from the same data, which are used to validate other areal products, for example, outputs of satellite precipitation algorithms and climate models, which are properly validated against unbiased, areally averaged in situ estimates of quantifiable uncertainty. For example, the standard error of a block kriging (Isaaks and Srivastava 1989) estimate is less than largest standard error of a point estimate produced by ordinary kriging within the block (area over which estimate applies) in question. Given appropriate statistical assumptions, unbiasedness can be shown, and uncertainty computed, theoretically, but both must be validated empirically.

Incorporating estimates derived from remote sensing platforms can reduce the uncertainty of rainfall estimates. Peer-reviewed literature abounds with examples of kriging being employed to merge radar rain estimates and rain gauge data, beginning with Krajewski (1987). More recent papers include those of Sideris et al. (2014) and Jewell and Gaussiat (2015), which gives an overview and comparison of several techniques and Park et al. (2017) and Verdin et al. (2015) which merge satellite and rain gauge data. It should be noted that all such schemes require knowledge of the error structure of the remote sensing algorithm output and cross validation to demonstrate the fidelity of the merged product, both of which necessitate comparisons with rainfall estimates traced solely to direct measurements at the surface. We will proceed with an eye to that necessity with the full understanding that the applicability of the resulting product will be limited, at times severely, by data sparseness and the highly variable nature of convective, tropical rainfall. We will begin by describing the rainfall gridding procedure in detail, and then demonstrate the reliability of the gridded product, through cross validation.

The point estimates generated using this method do not duplicate accumulation features on the scale of the grid spacing. They are intended to provide an easily accessible way to visualize historical distributions of sea level rainfall over the tropical Pacific (where available data allow) with quantifiable bias and uncertainty, and to be a tool for evaluating the reliability of areal rainfall estimates.

\section{Data selection and quality assurance}

Data for this study were drawn from the Pacific Rainfall (PACRAIN) database (Greene et al. 2008) for the period of January 1930-April 2018. These records originate from a variety of sources including the $\mathrm{Na}$ tional Centers for Environmental Information (NCEI), the New Zealand National Institute of Water and Atmospheric Research (NIWA), the French Polynesian Meteorological Service, the Schools of the Pacific Rainfall Climate Experiment (SPaRCE; Postawko et al. 1994), 
and the atlas of Pacific rainfall (Taylor 1973). These records can be obtained via web query at the PACRAIN website (www.pacrain.ou.edu), as individual files containing daily or monthly rainfall totals for fixed locations.

For this research, data sparseness is exacerbated by the need to exclude observations taken at locations where topography and/or differential heating of land and sea may lead to local, persistent spatial trends in rainfall, for example, due to orographic and sea breeze effects. We will hold to the postulate, consistent with the findings of Lavoie (1963) and the application of Morrissey and Greene (1993), that rainfall observations collected at atoll locations are representative of rainfall occurring in open ocean conditions and are more similar in their distributions in space and time to those over the surrounding open ocean, compared to the distributions of rainfall at nonatoll stations. Therefore, only rainfall observed at atoll locations are used to make gridded estimates, as the vast majority of gridpoint locations are oceanic, where persistent spatial rainfall trends would not be due to fixed variations in dynamic and thermodynamic forcing.

A discussion of the physical characteristics of the observed rainfall data (e.g., the intensities and spatial and temporal scales of features contributing to observed accumulations, and their organizing structures) and the implications of those characteristics on the interpolation process is not beyond the scope of this work, but is necessarily cursory. Deducing the detailed physical characteristics of individual rainfall features and, in turn, their specific contributions to rainfall totals is not generally possible for the spatial-temporal domain in question, due to insufficient data density in space and time.

In general, tropical Pacific rainfall comes mostly as a result of convective features, which vary greatly in prevalence, size, and characteristic accumulations. These features have horizontal scales as small as $1 \mathrm{~km}$ and can be seen in satellite imagery distributed individually and in larger organizing structures, more or less homogeneously, over regions with radii exceeding $1000 \mathrm{~km}$. Thus, daily rainfall over tropical oceans, even without tropical cyclones or other larger-scale rainfall features, can exhibit fluctuations on scales not typically sampled over ocean basins $(\sim 1-10 \mathrm{~km})$ that may approach, in magnitude, variations occurring over scales two to three orders of magnitude greater. Figure 1, derived from August 1971 atoll rainfall data within the PACRAIN database, for stations separated by less than $2000 \mathrm{~km}$, shows differences in monthly rainfall observations between atoll stations. From the historical example given in this figure it is clear that differences observed at separations less than $100 \mathrm{~km}$ may also be of the same order of magnitude as those typically observed between stations separated by much larger distances $(>1000 \mathrm{~km})$.

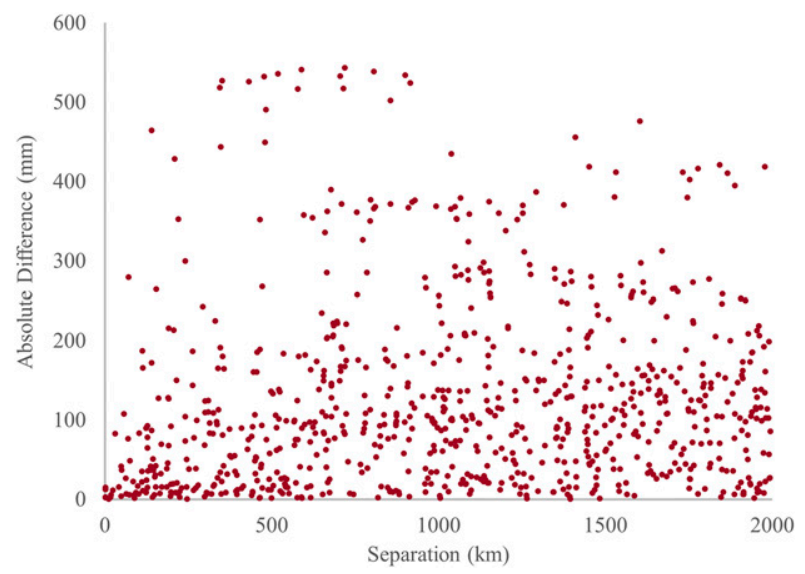

FIG. 1. Absolute monthly rainfall differences for stations separated by $<2000 \mathrm{~km}$. Derived from August 1971 atoll rainfall data contained in the PACRAIN database.

That differences in monthly rainfall between stations exhibit a very broad scatter and (weakly) more so with increasing separation distance should be expected. Monthly tropical rainfall totals are sums of accumulations due to meteorological features on a broad continuum of spatial scales, including individual showers and thunderstorms, convective storm clusters, tropical waves, tropical cyclones, the intertropical convergence zone (ITCZ), and the Madden-Julian oscillation (MJO). But the larger features include, or are entirely composed of, convective cells.

The following steps were taken to assure data quality, data comparability (internally), consistency relative to the domain over which the gridded product is meant to be applicable, and consistency of the data's sample probability density function with a Gaussian function:

- Only rainfall observed at Pacific atoll locationsdepicted in Fig. 2-were selected for this study. As noted in section 1, we take the position, consistent with Lavoie (1963) and Morrissey and Greene (1993), that rainfall at such stations is most consistent with that over the surrounding open ocean regions.

- Trace observations were changed to $0.0 \mathrm{~mm}$. With accumulations reported as small as $0.1 \mathrm{~mm}$ and typical monthly totals on the order of $10^{1}-10^{2} \mathrm{~mm}$, the error associated with zeroing a trace report is at least two orders of magnitude smaller than a typical monthly rainfall total or typical kriging standard error.

- Where quality assurance (QA) flags indicated that rains in one accumulation period were recorded in a subsequent accumulation period, for consecutive accumulation periods in different months, and where the smaller of the two periods was $24 \mathrm{~h}$ or less, accumulations were prorated between adjacent periods based on a constant accumulation rate over the aggregate 


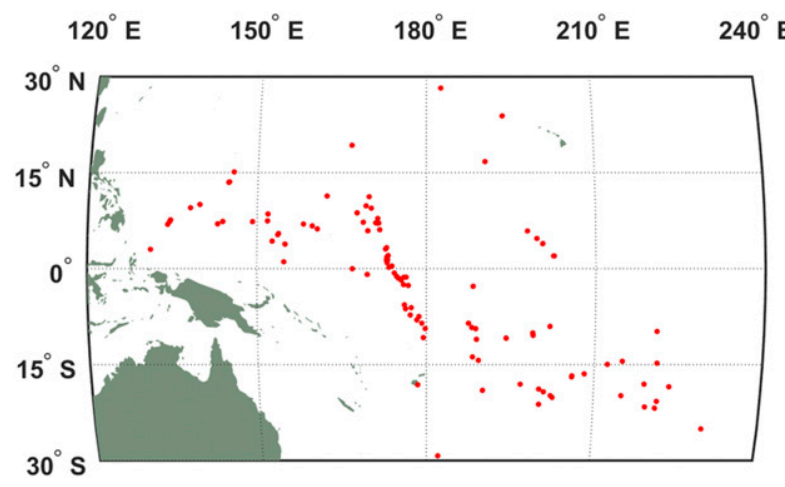

FIG. 2. Locations of atoll stations with records in the PACRAIN database.

period. Where the shorter period was greater than $24 \mathrm{~h}$, rain for both periods were discarded (flagged as missing and omitted from the gridding procedure). Monthly rainfall totals were also discarded where rainfall was reported as missing for at least one accumulation period for the month in question.

- Rainfall for stations with observation times different than 0000 UTC was prorated between consecutive months based on a constant accumulation rate during the accumulation period encompassing 0000 UTC.

- For stations with accumulation periods of less than a month (e.g., reported on a daily basis), accumulations were aggregated into monthly totals.

- Some cases of apparent duplicate records exist within the PACRAIN database. Notes, including a list of suspected duplicate stations, are provided with the results of data queries submitted to the PACRAIN website (www.pacrain.ou.edu). Records were deemed to be duplicates if 1) latitude and longitude metadata were identical and 2) station separation based on available metadata was greater than zero but less than $20 \mathrm{~km}$ and the root-mean-square difference in corresponding monthly rain records was less than $10 \mathrm{~mm}$. Where stations were found to be duplicates based on these criteria, a single station record was generated and assigned to the location with the longest period of record. In combining the records of suspected duplicates, $0.0-\mathrm{mm}$ values for one station were replaced with a positive value from the other station, where available. Otherwise, records for the station with the longest record were supplemented-missing values were replaced - with data from its assumed duplicate.

- Outliers, defined as observations that 1 ) deviated from an initial kriging estimate by a $z$ score of at least 5 , relative to the kriging standard error, and 2) were not within the range of the data used to produce that estimate, were removed. The rationale and methodology for eliminating these outliers is described below.
Spurious data must be identified in the context of the properties of tropical, convective rainfall. Monthly tropical rainfall totals are sums of accumulations due to meteorological and climatological features on a broad continuum of spatial and temporal scales. Consequently, extreme values may be due to local superposition over time of features on any one or all of these scales. Based only on the examples of station-to-station differences shown in Fig. 1, the threshold used here for omitting data from the estimation process must consider the real possibility of stations separated by $100 \mathrm{~km}$ exhibiting differences in monthly rainfall of more than $100 \mathrm{~mm}$. Without supplemental information (e.g., from a satellite or radar), finer-scale in situ sampling in space and time, or notation in the database suggesting a data quality issue, discarding extremes demands manual examination of the surrounding data to exclude the possibility of meteorological phenomena, unsampled by the stations used to estimate rainfall but affecting the station reporting the anomalous value.

We defined outliers to be removed as observations that 1) deviated from an initial kriging estimate by a $z$ score of at least 5, relative to the kriging standard error, and 2) were not within the range of the data used to produce that estimate. These outliers were examined manually and, in all cases, they proved inconsistent with multiple observations within $200 \mathrm{~km}$ of, and in widely differing directions from, the location of the outlying report. Furthermore, of these anomalies, none could be explained in terms of meteorological phenomena on scales smaller than that in the local group of stations, such as a small tropical cyclone, or in terms of a small number of large daily totals that could be the natural, if infrequent, result of "random" differences in the number of convective rainfall events affecting the local group of stations over the course of a month. Of the 20 reports meeting the outlier criteria, 7 were found at one station. A manual examination of that station's records indicated other instances of rainfall values seemingly at odds with those reported by the local group of stations; its entire record was excluded from the estimation process. The remaining 13 outlier reports were deemed to be isolated and were excluded individually.

Following the steps to assure quality and consistency, a square root transform was applied to rainfall observations. The square root transform was shown (for the case of daily rainfall) in Schuurmans et al. (2007) to yield transformed quantiles more similar to those of the Gaussian distribution than those resulting from a logarithmic transform. This transform is also used in Sideris et al. (2014) for both radar estimates and rain gauge observations prior to kriging. The transform is adopted here for three reasons. First, it results in a symmetric 
January

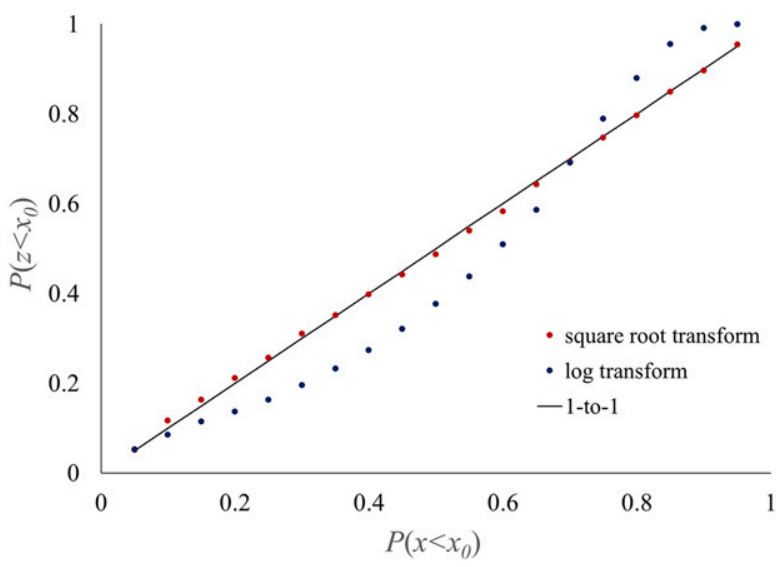

July

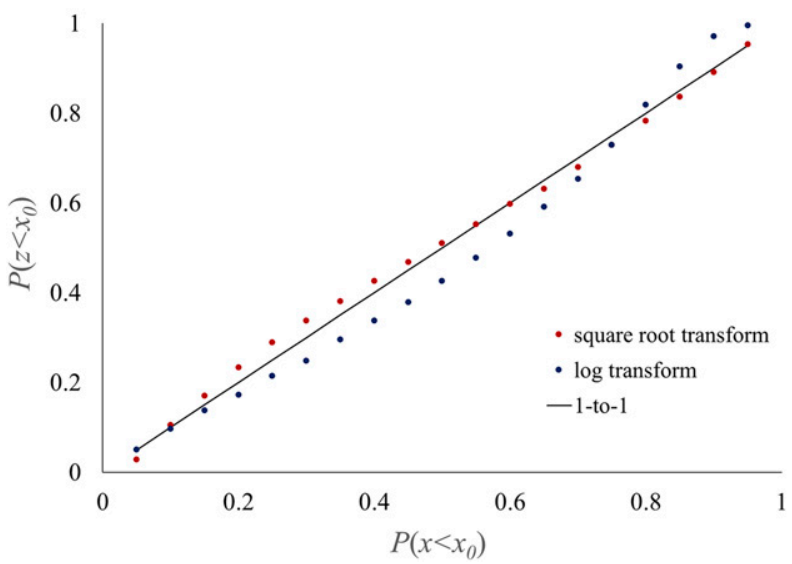

April

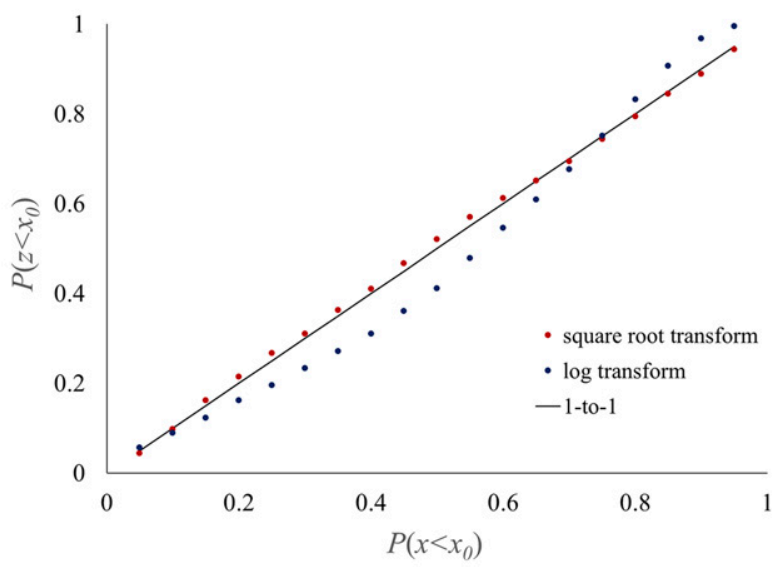

October

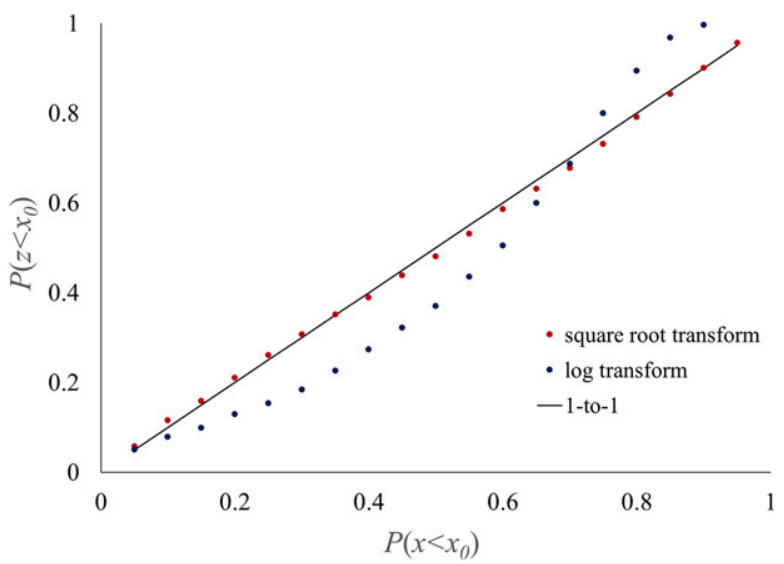

FIG. 3. $P-P$ plots of square root and log-transformed rainfall vs Gaussian model derived from PACRAIN atoll data (1930-2018 for January-July, 1930-2017 for August-December).

distribution. Ordinary kriging produces estimates of reduced variance. This variance reduction corresponds to reduced probabilities (relative to the data) of relatively high and low values. Such distributional differences are not symmetric when the data are skewed. Second, it allows for simple estimation of probability thresholds based on kriging variance. Finally, the square root transform provides for a simple, unbiased back transformation. Figure 3 shows $P-P$ plots for transformed monthly rainfall versus Gaussian distributions of the same means and standard deviations, for $\log$ and square root transforms. As with the $Q-Q$ plots given by Schuurmans et al. (2007) comparing the $\log$ and square root transformations, it can be seen that square root-transformed rainfall is more similar in distribution than log-transformed rainfall to a Gaussian variable of the same mean and standard deviation. Probabilities are decidedly asymmetric for the log transform, being concentrated to the right of the mean. In short, the log transform visibly "overcorrects" for the expansive right tail observed in the dataset.

\section{Semivariogram estimation}

Estimation of semivariograms began with computation of semivariance data $\hat{\gamma}$. These data were computed, as shown in (3.1), for each month $m$ at integer $k$ multiples of $50 \mathrm{~km}$ of separation, by applying normalized weights given by a Gaussian function to squared differences between transformed rainfall values $z$ at stations $i$ and $j$, separated by great circle distances $h_{i j}$, for month $m$ and year $y$. The interval of $50 \mathrm{~km}$ between semivariance data points was chosen subjectively, but with attention to the minimal sensitivity of semivariogram parameters (and, in turn, estimation error) to that distance (and to the Gaussian standard deviation) from 20 to $100 \mathrm{~km}$ : 


$$
\hat{\gamma}_{m}(50 k)=\frac{1}{2} \frac{\sum_{i=1}^{n-1} \sum_{j=i+1}^{n} \sum_{y_{\text {valid }}} g\left(50 k-h_{i j}\right)\left(z_{i m y}-z_{j m y}\right)^{2}}{\sum_{i=1}^{n-1} \sum_{j=i+1}^{n} \sum_{y_{\text {valid }}} g\left(50 k-h_{i j}\right)} .
$$

The year summation is for valid years $\left(y_{\text {valid }}\right)$, that is, years in which both $z_{i m y}$ and $z_{j m y}$ are valid. The Gaussian function $g(x)$ in (3.1) is given by

$$
g(x)=\frac{1}{\sqrt{2 \pi \alpha^{2}}} e^{-x^{2} / 2 \alpha^{2}},
$$

where $\alpha=50 \mathrm{~km}$. The weight function (3.2) is not bounded, which results in some smoothing, and semivariance estimates that are not independent.

Note that the adoption of ordinary kriging is not logically consistent with computing semivariance without the assumption of second-order stationarity. Universal kriging provides a logical alternative, assuming nonstationarity; however, the spatial trend model must be chosen-and to be consistent, applied in the computation of semivariance-which (among other things) makes incorporating data from multiple years in the semivariogram estimation procedure a dubious exercise. Furthermore, and as suggested by Isaaks and Srivastava (1989) and Wackernagel (2003), universal kriging is not a robust method with respect to estimates made outside the spatial interior of the group of stations used for estimation due to extrapolation of trends.

The formulation of semivariograms without accounting for nonstationarity is not accepted lightly; however, of greater concern is that kriging standard errors are demonstrated to be predictive of actual standard errors via cross validation and expected to retain their predictive qualities at locations far removed from estimator stations. The latter cannot be maintained with respect to universal kriging to the degree it can with respect to ordinary kriging, due to the extrapolation problems mentioned previously.

It should be noted that average spatial trends in the rainfall field serve to increase semivariance values (3.1) for lags over which they persist. This can degrade the fidelity of the correlation structure expressed by the associated semivariogram, the parametric form of which also directly affects the expressed correlation structure.

An isotropic exponential semivariogram model (3.3) was fit to the semivariance data described previously. See Cressie (1985, 1993b, chapter 2) and Chiles and Delfiner (1999, chapter 2) for information on common semivariogram models and fitting techniques:

$$
\gamma(h)=\gamma_{0}+s\left(1-e^{h / \tau}\right),
$$

where $h, \gamma_{0}, s$, and $\tau$ represent isotropic separation, nugget, sill, and range, respectively. Nugget, sill, and range parameters were found via iteration to obtain minimum squared residues between the model and semivariance data generated for each month. Data from many years are used to estimate a single variogram for each of the 12 calendar months.

For the purposes of cross validation, each stations' data were omitted sequentially from the estimation process for the semivariograms used in estimating its monthly rainfall history. For the gridding procedure, all available data were used.

Figure 4 shows model semivariograms together with the semivariance data to which they are fit for the months of January, April, July, and October-months meant to give a sampling of the seasonal variability of semivariograms. The semivariograms of Fig. 4 are those used to produce gridded estimates, being derived from all stations. There are too many semivariograms used in the cross-validation procedure to show (one for each month for each station). A noteworthy feature seen in the plots of Fig. 4 is the nearly factor-of-2 change in computed sill between January and July. This is likely due in part to differences in the geometrical relationships between the spatial distribution of stations and average rainfall gradients for the two months; that is, a difference in relative sampling probably accounts for some of the difference in the semivariograms. This position is supported by Fig. 5. A cursory inspection shows that the entire range of means in January is accommodated in approximately the maximum separation considered $(2000 \mathrm{~km})$ in fitting the semivariograms; it is accommodated in roughly twice that distance in July. The apparent shift seen in all four months depicted in Fig. 4, in the trend of the semivariance data at approximately $800 \mathrm{~km}$ is also of interest. The isotropic exponential model was retained despite this appearance due to several considerations: limiting optimization to 3 degrees of freedom due to sample size; its continuous natureit seems implausible to the authors that the expectation of squared differences would reach a hard maximum at a particular distance; the considerable likelihood that the transition's persistence (which is not unique) is due, in part, to the peculiarities of the available spatial sampling; and its simple interpretation and prevalent adoption in the literature.

It should be noted that data sparseness can affect the fit of model semivariogram parameters. Exponential semivariograms fit to these data, where few stations are separated by distances of, or less than, the scale of the smallest rainfall features, may exhibit larger nuggets 


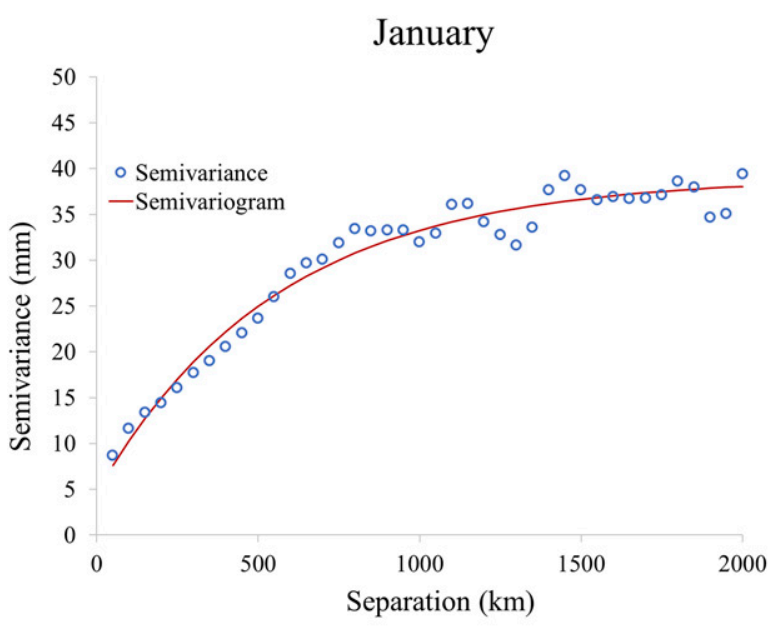

July

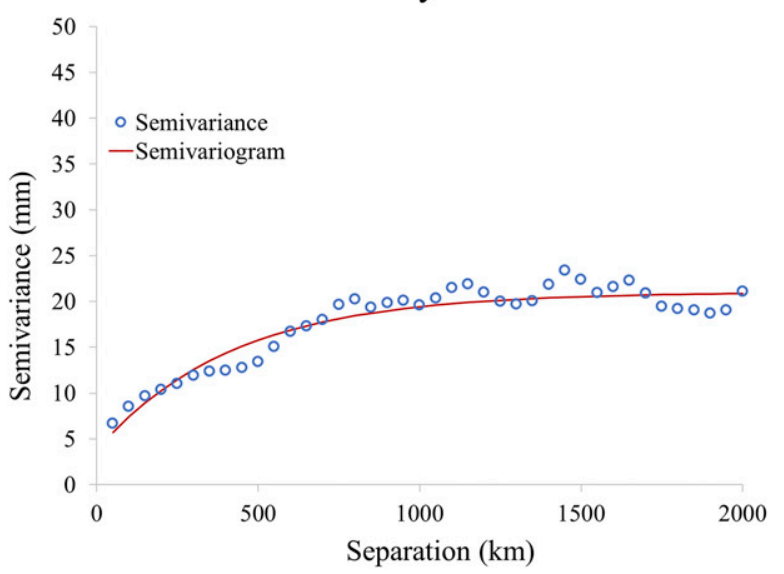

April

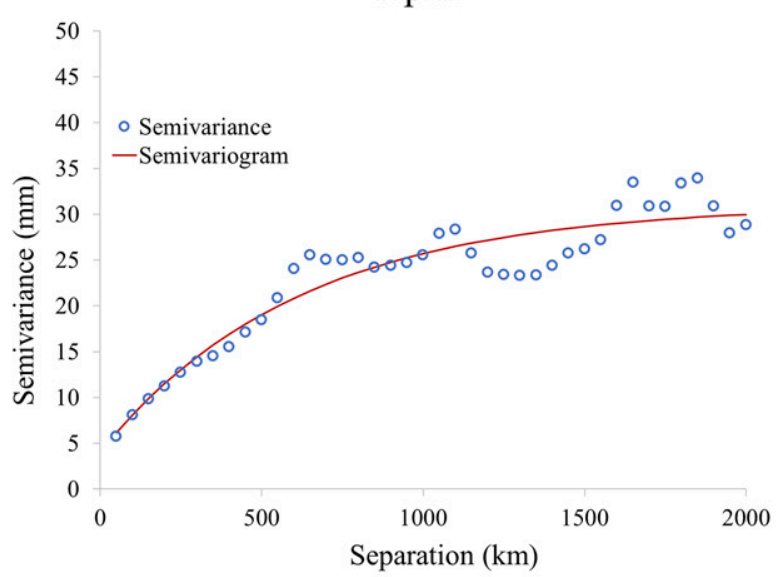

October

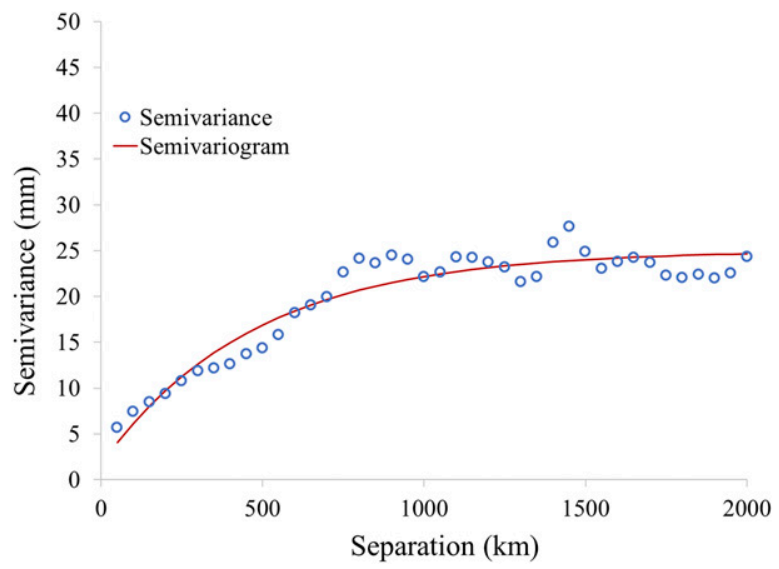

FIG. 4. Model semivariograms derived from PACRAIN atoll data (1930-2018 for January-July, 1930-2017 for August-December).

and ranges relative to those based on denser data. With regard to kriging, the practical effect of a larger nugget is increased smoothing and a larger minimum estimation standard error, for a given number of estimator stations, everywhere the variogram is applied (except where an estimate is collocated with an estimator station). The practical effect of an increased range is increased smoothing of estimates-less difference in weights applied to a given set of observations.

\section{Estimation procedure}

Rainfall estimates for cross validation and gridding were produced using the same implementation of ordinary kriging. Kriging systems (4.1) were populated by evaluating the model semivariograms (3.3):

$$
\left[\begin{array}{cccc}
\gamma\left(h_{11}\right) & \cdots & \gamma\left(h_{1 n}\right) & 1 \\
\vdots & \ddots & \vdots & \vdots \\
\gamma\left(h_{n 1}\right) & \cdots & \gamma\left(h_{n n}\right) & 1 \\
1 & \cdots & 1 & 0
\end{array}\right]\left[\begin{array}{c}
w_{1} \\
\vdots \\
w_{n} \\
\mu
\end{array}\right]=\left[\begin{array}{c}
\gamma\left(h_{1 p}\right) \\
\vdots \\
\gamma\left(h_{n p}\right) \\
1
\end{array}\right]
$$

In the equations above, $\gamma\left(h_{i j}\right)$ are variogram model values for distances separating stations $i$ and $j$ within a group of $n$ estimator stations and $\gamma\left(h_{i p}\right)$ are the variogram model values for distances separating estimator stations $i$ from the station, or grid node $p$ where rain is to be estimated. Solution of (4.2) yields the set of weights $w_{i}$ and the Lagrange parameter $\mu$ with which estimates of the transformed variable $\hat{z}_{p}$,

$$
\hat{z}_{p}=\sum_{i} w_{i} z_{i}
$$



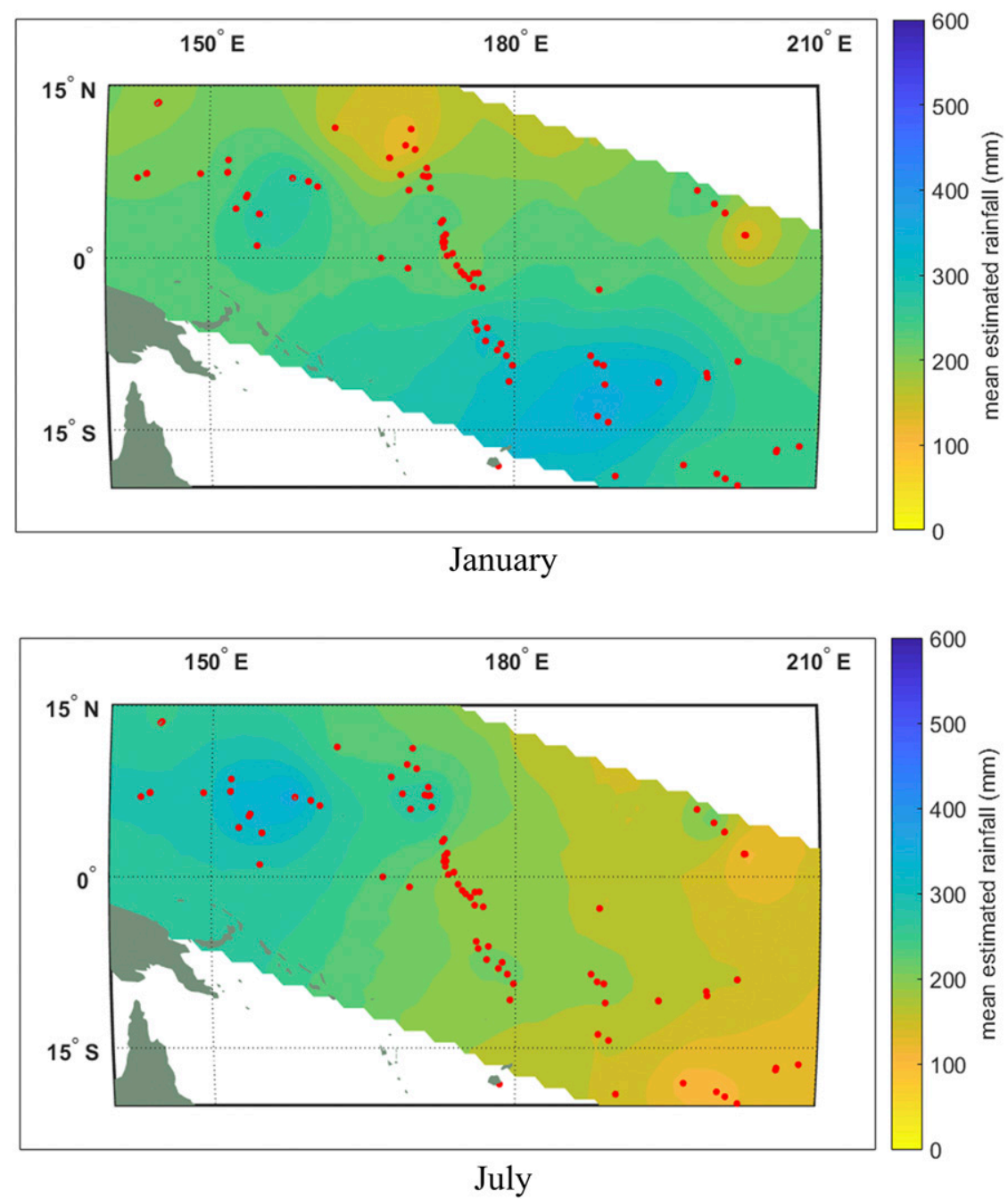

FIG. 5. Mean estimated rainfall for January and July derived from PACRAIN atoll data (1930-2018 for January-July, 1930-2017 for August-December.

and the ordinary kriging variance $\sigma_{\mathrm{OK}}^{2}$ are computed:

$$
\sigma_{\mathrm{OK}}^{2}=\sum_{i} w_{i} \gamma\left(h_{i p}\right)+\mu .
$$

Clear derivations of the ordinary kriging system can be found in Isaaks and Srivastava (1989, chapter 12) and also in Wackernagel (2003, chapter 11).

Rainfall was computed for station locations and grid nodes using groups of $n=9$ estimator stations drawn from the set of 12 closest stations to the location in question. The number of estimator stations was chosen based on standard errors and biases computed in exploratory trials. As seen in Fig. 6, standard errors change much less fractionally for $n>6$ relative to $n<6$, while Fig. 7 indicates that bias is minimized in the aggregate for $n=9$. The bias shown in Fig. 7 reflects the combined effects of all sources of bias and can be made objectively small-at least two orders of magnitude smaller than mean monthly rains.

Bias appearing in the back-transformed rainfall estimates $\hat{r}$, given by Sideris et al. (2014),

$$
\hat{r}=\hat{z}^{2}+\sigma_{\mathrm{OK}}^{2},
$$

can arise from both the transformed estimates themselves and from the kriging variance of the transformed variable. This is not to say that (4.4) is a biased estimator; bias can arise from the correct mapping of transformed estimates which do not have the variance of transformed observations (due to smoothing characteristic of a linear, unbiased estimator) and from kriging variance estimates that are based on semivariograms that are computed globally for each month.

For kriging, the reduction of variance at location $\mathbf{x}$ is given by Yamamoto (2000) as 


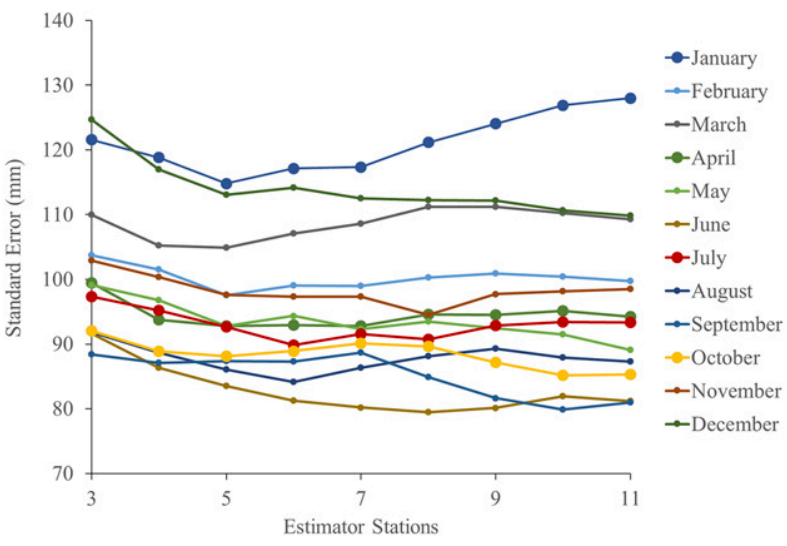

FIG. 6. Standard error of kriging estimates vs number of estimator stations derived from PACRAIN atoll data (1930-2018 for January-July, 1930-2017 for August-December).

$$
\operatorname{var}[z(\mathbf{x})]-\operatorname{var}[\hat{z}(\mathbf{x})]=\sigma_{\mathrm{OK}}^{2}+2 \mu \geq 0 .
$$

A postprocessing algorithm to reclaim the variance of the original data, given by Yamamoto (2005), applies successive corrections obtained by subtracting cross estimates obtained for the data points used from the data themselves, via kriging, to the grid node or crossvalidation point. The incorporation of this postprocessing scheme requires a theoretical estimator of kriging variance for the various levels of adjustment and demonstration that

$$
\sigma_{\mathrm{OK}_{l+1}}^{2} \leq \sigma_{\mathrm{OK}_{l}}^{2},
$$

where $l$ denotes the number of corrections applied. If (4.6) does not hold in general, then reclaiming the data variance comes at the potential cost of increased standard error, to be determined empirically. Thus, since the scheme may increase standard error, and (4.6) is not shown theoretically, we have not included this approach. Potential alternatives to the algorithm of Yamamoto (2005) include constrained kriging (Cressie 1993a) and covariance-matching constrained kriging (Aldworth and Cressie 2003).

As for bias due to the kriging variance of the transformed variable, in adopting global semivariograms it is assumed that rainfall fields will exhibit the same values of semivariance at the same spatial lags, for all instances of a given month, regardless of local means or the prevailing mean of a given instance of that month. The back transform assumes symmetry and homoscedasticity in the error distribution for $\hat{z}$.

The method used to ensure only nonnegative estimates is less restrictive than that proposed by Barnes and Johnson (1984) or Szidarovszky et al. (1987) which enforce positive weights, although the latter also aims

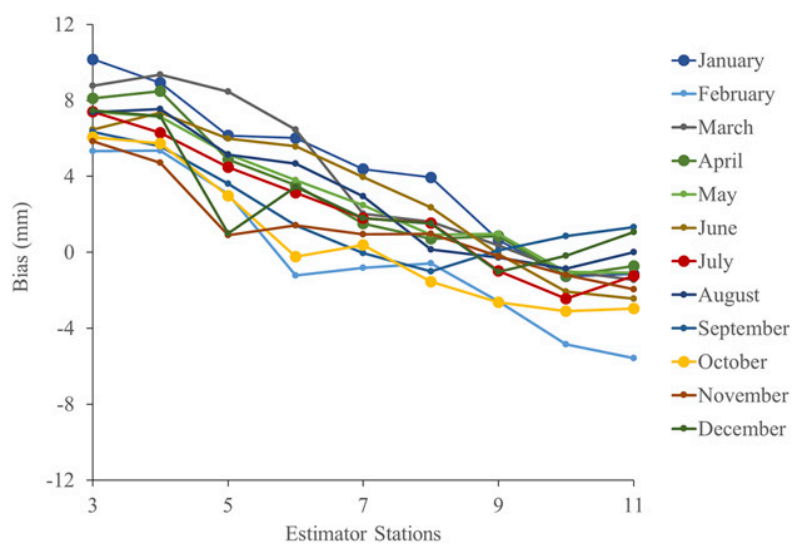

FIG. 7. Bias of kriging estimates vs number of estimator stations derived from PACRAIN atoll data (1930-2018 for January-July, 1930-2017 for August-December).

to obtain the least uncertain estimate as judged by the corresponding value of $\sigma_{\mathrm{OK}}^{2}$. We simply retained nonnegative rainfall estimates of minimum kriging variance. While negative rains are distinctly unphysical, negative weights as applied to rainfall estimation are no more unphysical than the notion that rainfall at a point can be approximated as a linear combination of rains observed in a spatial neighborhood of that point. Still, the presence of negative weights can also result in estimates well outside the range of rainfall observations in the neighborhood. Such estimates $\hat{r}$ were excluded where

$$
\frac{\left(\hat{r}-\bar{r}_{\text {local }}\right)}{\sigma_{\text {local_obs }}}>5,
$$

in which $\bar{r}_{\text {local }}$ is the mean of the observations used in estimation and $\sigma_{\text {local_obs }}$ is the standard deviation of those observations.

\section{Cross-validation results}

For the study period of 1930-2018, there were 7672 monthly observations in the atoll dataset for which estimates could be derived using the best 9-station neighborhood out of 12 closest stations criteria. Regressions of estimated and observed rainfall for a sampling of four seasonally representative months are shown in Fig. 8.

Goodness of fit, as judged by the $R^{2}$ metric, varies from its maximum in February (0.657) to its minimum in November (0.524). Slope varies by approximately $8 \%$ from its April value of 1.0057 for April to 0.9228 in August. The $R^{2}$ results are inevitably related, to some degree, to the spatial distribution of stations addressed in cross validation and cannot be taken as indicative 

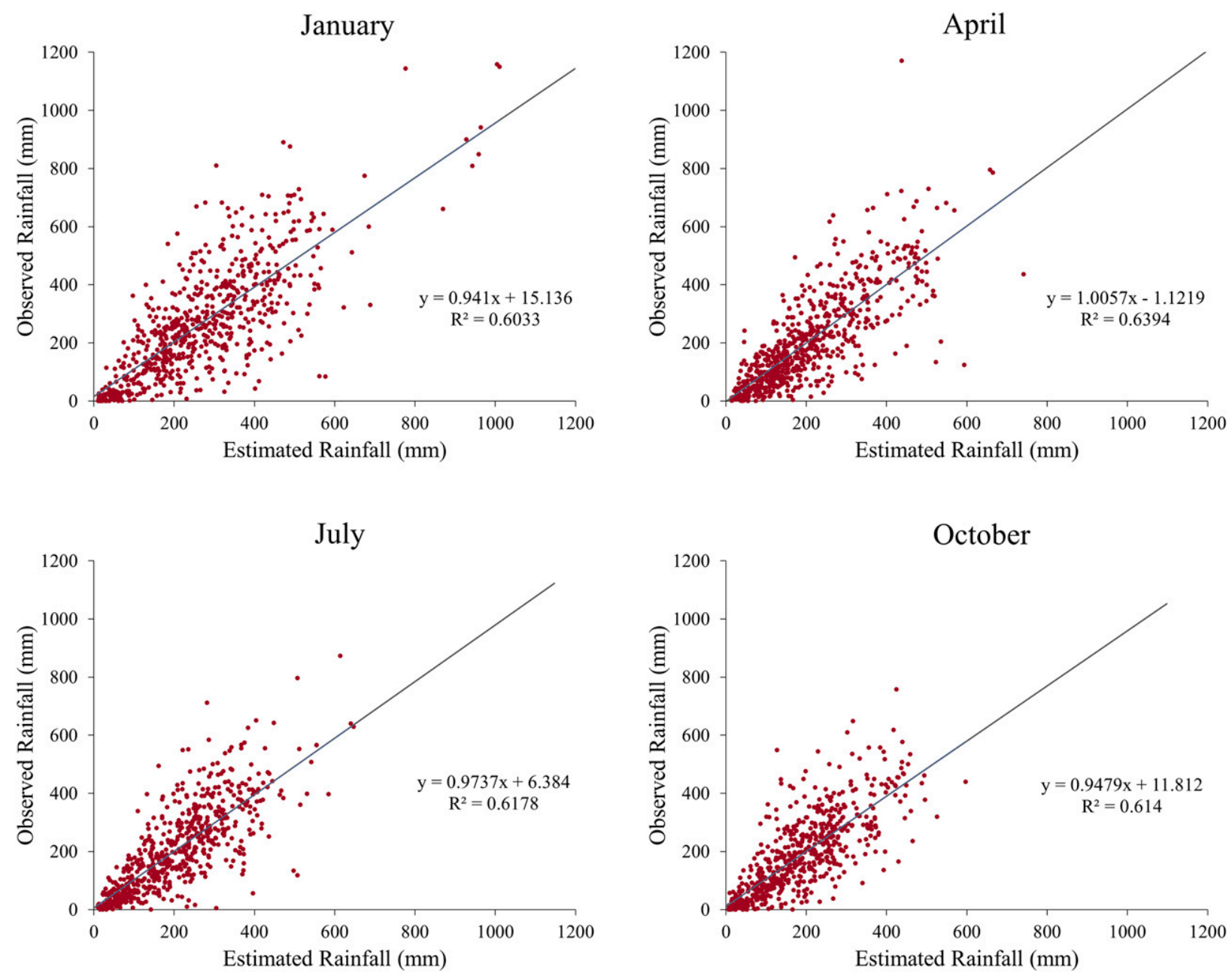

FIG. 8. Scatterplots of cross-validation estimated and observed rainfall derived from PACRAIN atoll data (1930-2018 for January-July, 1930-2017 for August-December).

of results for the grid domain, in general. However, $R^{2}$ and slope exhibit durability with respect to, for example, restriction to halves of the geographic domain.

A set of $P-P$ plots in Fig. 9 compares sample cumulative probabilities for estimated and observed rains, where $P_{\text {est }}\left(\hat{z}<\hat{z}_{0}\right)$ and $P_{\text {est }}\left(\hat{r}<\hat{r}_{0}\right)$ are sample probabilities of transformed and back-transformed crossvalidation estimates, respectively, and $P_{\mathrm{obs}}\left(z<\hat{z}_{0}\right)$ and $P_{\text {obs }}\left(r<\hat{r}_{0}\right)$ are the sample probabilities of transformed and nontransformed observations, respectively. The reduction in variance is seen in estimate probabilities mapping to a narrower range of observation probabilities (e.g., there are more observations below $50 \mathrm{~mm}$ than there are estimates, and more observations above $400 \mathrm{~mm}$ than there are estimates). This reduction in variance of estimates, relative to observations, an expected feature of an unbiased, linear estimator, is seen in both transformed and back-transformed estimates.

Overall negative bias, which could result (in isolation) from the back transform of a set of estimates of reduced variance is not generally evident in Fig. 7 except for estimator groups of $n>9$. It is notable in Fig. 9 that the shift in observation probabilities under back transformation of estimates is, in all cases shown, toward higher cumulative probabilities, but is relatively small for high-end estimates.

The kriging variance, which is directly proportional to the sum of nugget and sill of the semivariogram employed, can be increased by the presence of relatively large, isolated rainfall totals in the datasets used to estimate semivariograms. A single set of semivariogram parameters is determined globally, and for all available years, for each calendar month. Kriging variance is determined identically for a particular semivariogram and configuration of estimator stations, regardless of 

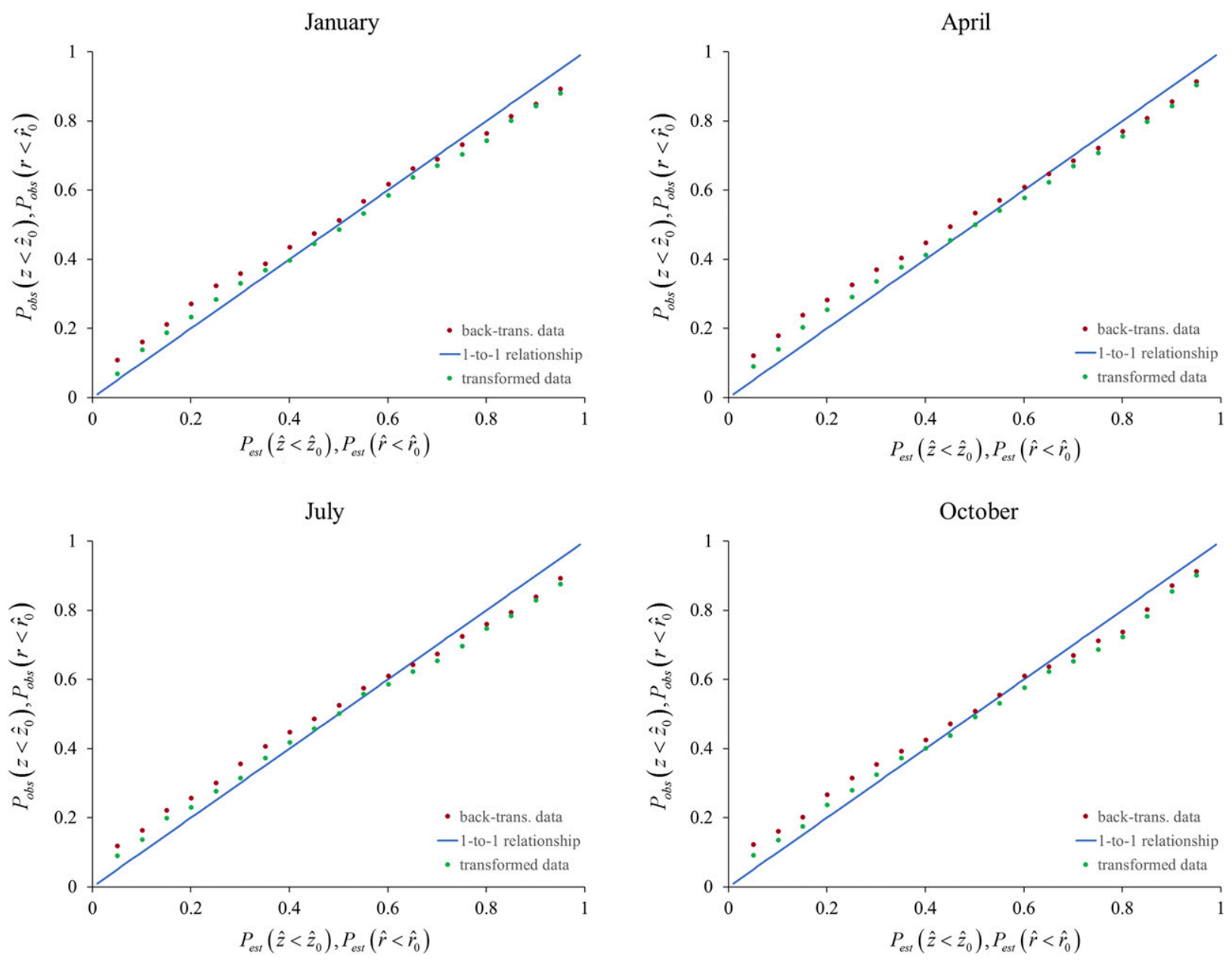

FIG. 9. $P-P$ plots of cross-validation estimated and observed rainfall samples derived from PACRAIN atoll data (1930-2018 for January-July, 1930-2017 for August-December).

the actual rainfall observations for those stations. Thus, where groups of estimator observations show relatively low variability, the second term on the right of the back transformation (4.4) can result in a distributional shift to the right of estimates relative to observations.

Back-transformed kriging-estimated error variances were computed using the back transform given by Sideris et al. (2014) for the square root transform:

$$
\sigma_{\mathrm{BT}}^{2}=4 \mu^{2} \sigma_{\mathrm{OK}}^{2}+2 \sigma_{\mathrm{OK}}^{4} .
$$

Corresponding estimated standard errors are compared to observed standard errors $\sigma_{\mathrm{OB}_{i}}$ computed as follows using the unbiased formula for variance for 31 pairs of rainfall estimates and observations:

$$
\sigma_{\mathrm{OB}_{j}}^{2}=\frac{1}{30} \sum_{i=j-15}^{j+15}\left(\hat{r}_{i}-r_{i}\right)^{2}
$$

where $\hat{r}_{i}$ and $r_{i}$ are sorted by $\sigma_{\mathrm{BT}}$ and $j=31 k-16, k$ being an integer such that $i$ is neither negative nor greater than the number of available data pairs generated in cross validation for a particular calendar month. Plots of estimated and observed standard errors are shown in Fig. 10. The data points used for these plots are independent, in that they contain no common pairs of estimates and observations. The number of data points chosen for computing variances is the first odd number (for the sake of symmetry) exceeding 30 , which is the commonly accepted minimum sample size for approximate confidence thresholds to be derived from a normal distribution.

Two things are immediately apparent in the plots of Fig. 10. First, in all cases, a least squares regression of the data suggests that estimated standard errors increase more rapidly than observed standard errors. Second, the plots suggest (for January, July, and October) that a linear relationship is applicable to 

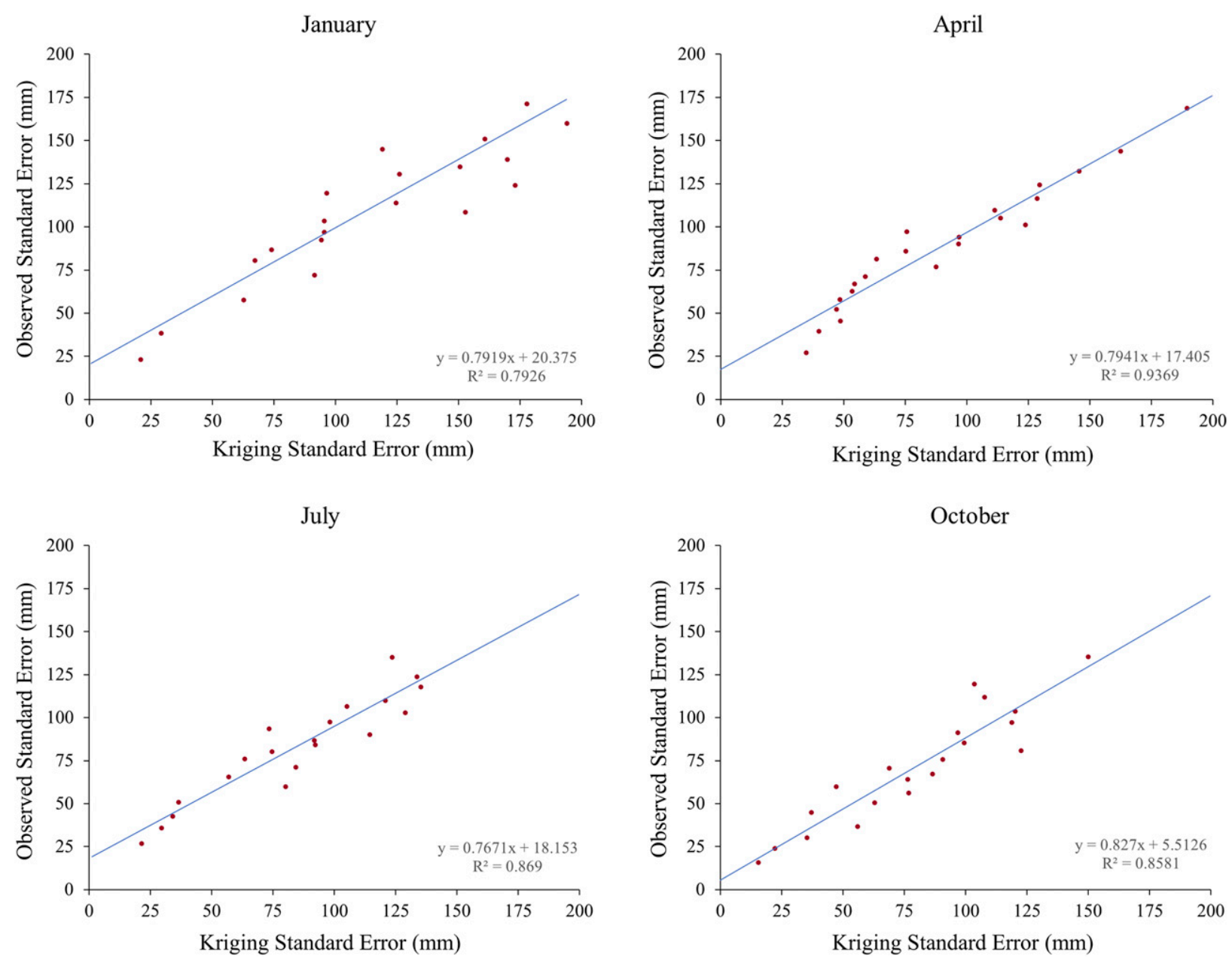

FIG. 10. Standard errors-kriging estimated (back transformed) vs observed derived from PACRAIN atoll data (1930-2018 for January-July, 1930-2017 for August-December).

estimated and actual standard errors, even though data used to compute observed standard errors are selected based on estimated standard errors which, in turn, are wholly dependent on semivariograms and the spatial distributions of estimator stations used. In other words, data for computing "observed" error variance are not sampled in a spatially homogeneous way. Nevertheless, theoretical standard errors are shown to be predictive of actual standard errors.

\section{Gridded monthly rainfall products}

For each node of the $1^{\circ} \times 1^{\circ}$ grid encompassing the atoll stations used in this paper, the following parameters were computed:

$$
\begin{aligned}
& \hat{r}=\hat{z}^{2}+\sigma_{\mathrm{OK}}^{2}: \text { mean rain estimate }(\mathrm{mm}) \\
& \sigma_{\mathrm{BT}}^{2}=\left(4 \hat{z}^{2} \sigma_{\mathrm{OK}}^{2}+2 \sigma_{\mathrm{OK}}^{4}\right)^{1 / 2}: \text { standard error of back-transformed estimates }(\mathrm{mm}) \\
& \sigma_{\mathrm{OK}}: \text { standard error of transformed estimates }\left(\mathrm{mm}^{1 / 2}\right) .
\end{aligned}
$$

Kriging standard error for the transformed estimates is included in the web-published products to facilitate the computation of confidence bounds based on a
Gaussian error distribution. For example, upper- and lower-quartile estimates can be derived from the published data via 


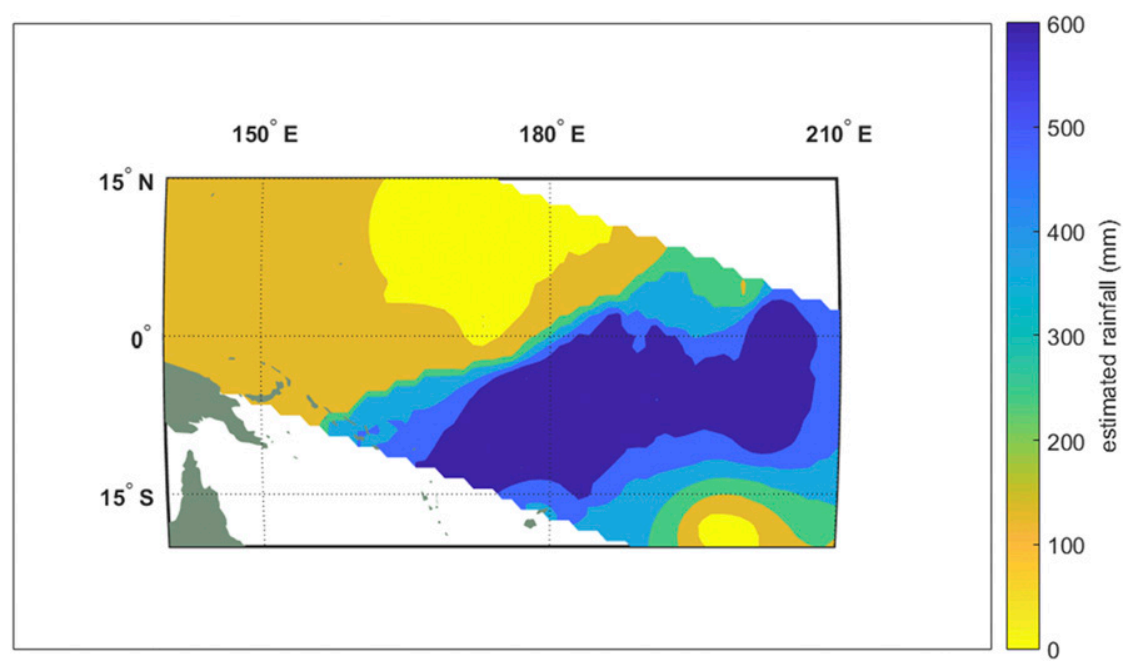

FIG. 11. Kriging-estimated rainfall for January 1983 (El Niño).

$$
\hat{r}_{\mathrm{lq}, \mathrm{uq}}=\left[\left(\hat{r}-\sigma_{\mathrm{OK}}^{2}\right)^{1 / 2} \pm 0.6745 \sigma_{\mathrm{OK}}\right]^{2},
$$

where the number 0.6745 corresponds to the half-width ( $z$ score) of the interval between the 25th and 75th percentile thresholds of the standard normal distribution. These back transforms are given Sideris et al. (2014) for the square root transformation. It should be noted that the validity of (6.1) and (6.2) hinge on the distributional similarity of the estimates and a Gaussian distribution of the same mean and variance.

The method for generating gridded estimates deviated from that used in cross validation in two ways: first, no observations were excluded from the pool of data from which estimator neighborhoods were drawn, and second, estimates were still made in cases where there were less than 12 available observations. In the latter case, neighborhoods of nine stations were simply drawn, where available, and selected based on the criteria explained in section 5. The former deviation is a natural step, as the reason for excluding stations in the estimation process for cross validation is to ensure the integrity of comparisons between the data (excluded) and the estimate (constructed from the remainder of the data). The latter simply allows for gridded estimates in as many cases as possible without violating the applicability of the cross-validation results with regard to the number of estimator stations.

Plots of sample gridded rains for January 1983 and January 1989 are given in Figs. 11 and 12, respectively. These examples were chosen for their representativeness of interesting climate events: El Niño in case of January 1983 and La Niña in the case of January 1989. The less-than-smooth presentation is due to a combination of factors: the nature of the plotting software in conjunction with the $1^{\circ}$ resolution of the product and the use of local estimator neighborhoods rather than a global fit.

Two notable differences between the patterns seen in Figs. 11 and 12 and the "canonical" El Niño and La Niña patterns are the heavy rainfall totals extending southwestward toward the Solomon Islands and Vanuatu in January 1983 and the local rainfall maximum seen near $180^{\circ} \mathrm{E} / \mathrm{W}$ at approximately $10^{\circ} \mathrm{S}$ in January 1989 . The former is due to estimation where there is a lack of atoll observations, the latter to a monthly rainfall total of $600 \mathrm{~mm}$ (large, relative to observations in the vicinity). The daily rainfall observations associated with the local maximum in monthly rainfall of Fig. 12 are unexceptional and cannot be objectively excluded based on other monthly totals in the spatially sparse neighborhood.

For comparison, corresponding gridded monthly rainfall estimates of the Global Precipitation Climatology Project (GPCP), version 2.3, are shown in Figs. 13 and 14. One notable difference between Fig. 11 and Fig. 13 is seen in the rainfall gradient at approximately $175^{\circ} \mathrm{E}$, just south of the equator. While direct comparisons of the two products are not strictly valid due to the nature of the difference between point and areal estimators, any identified difference is not necessarily due to the difference between a point and areal estimators. Rainfall is sampled immediately north and south of the region of strong gradient, but stations sampling it are clustered along narrow bands. Standardized differences (see Fig. 15) based on kriging variance are relatively large to the east and west of the location in question. The situation is not unexpected, given that satellite data are available where there are no surface rainfall observations and that areal rainfall estimators relying heavily on satellite data may contradict surface observations, 


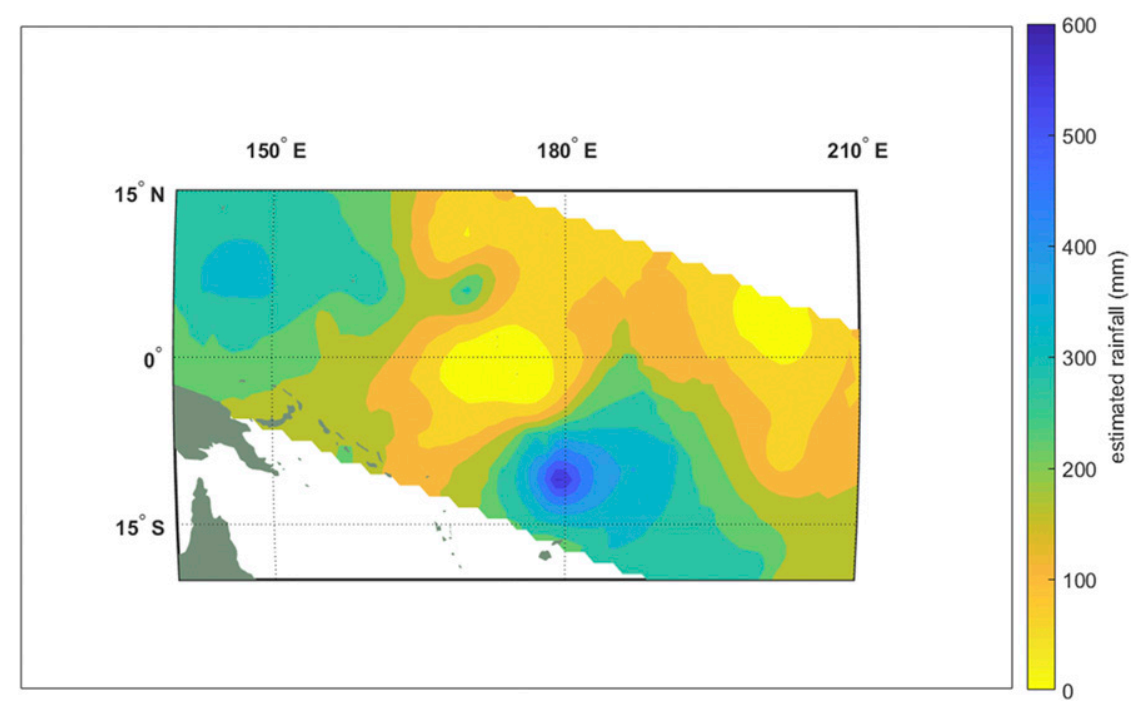

FIG. 12. Kriging-estimated rainfall for January 1989 (La Niña).

even if they are unbiased. For a description of the GPCP products and their derivation, see Adler et al. (2003, 2018). GPCP monthly estimates are merged products, derived using rain gauge, satellite, and sounding data.

To place the visible differences between the precipitation patterns exhibited by the kriging estimates and the GPCP estimates in perspective, standardized rainfall differences (GPCP - kriging) based on kriging standard error are plotted in Fig. 15. Standardized differences with magnitudes in excess of two are mainly confined to regions far removed from available atoll data, and where, based on well-known precipitation patterns associated with El Niño, estimates are made based on observations in an area that would be expected to see the statistical anomalies of the opposite sign. Care must be taken not to ascribe undue meaning to this comparison. The products have different resolutions and one is correctly interpreted as consisting of areal estimates (GPCP) - the other of point estimates.

\section{Summary}

Gridded historical rainfall estimates, based on monthly rainfall observed at atoll locations, have been produced for a portion of the tropical Pacific using ordinary kriging. The importance of these estimates is rooted in their unbiasedness and validated standard error estimates, as well as their availability for time periods prior to merged products, such as the GPCP monthly rainfall grids.

The data were chosen as being the most similar, in terms of rainfall climatology, to open ocean locations.

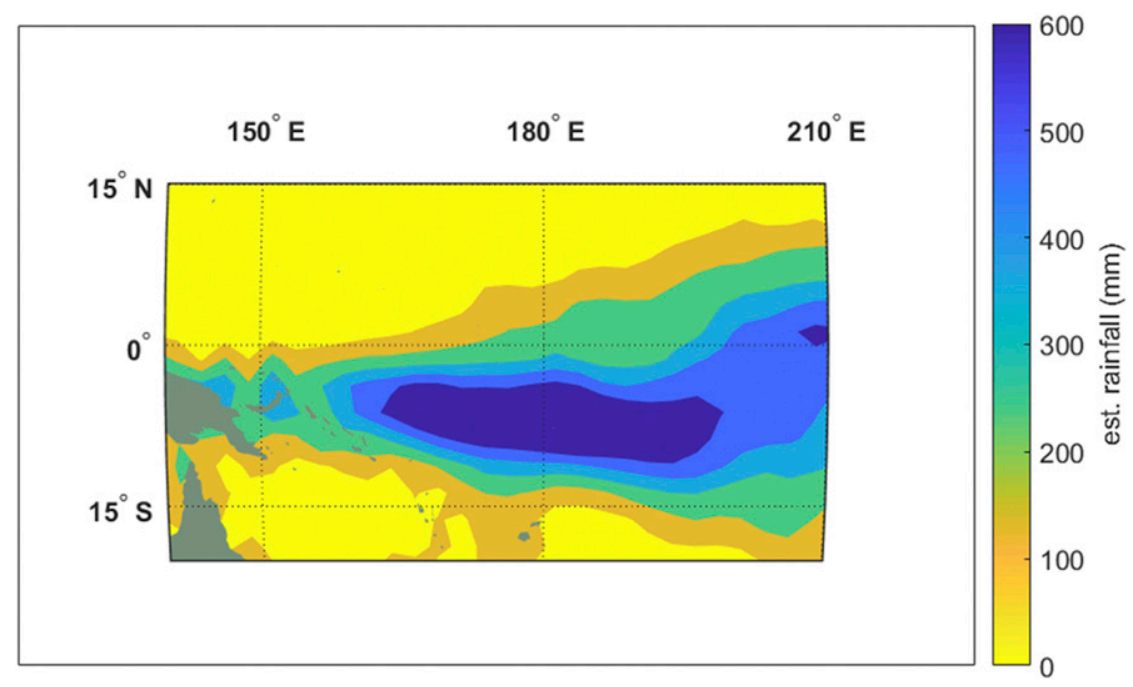

FIG. 13. GPCP rainfall estimates for January 1983 (El Niño). 


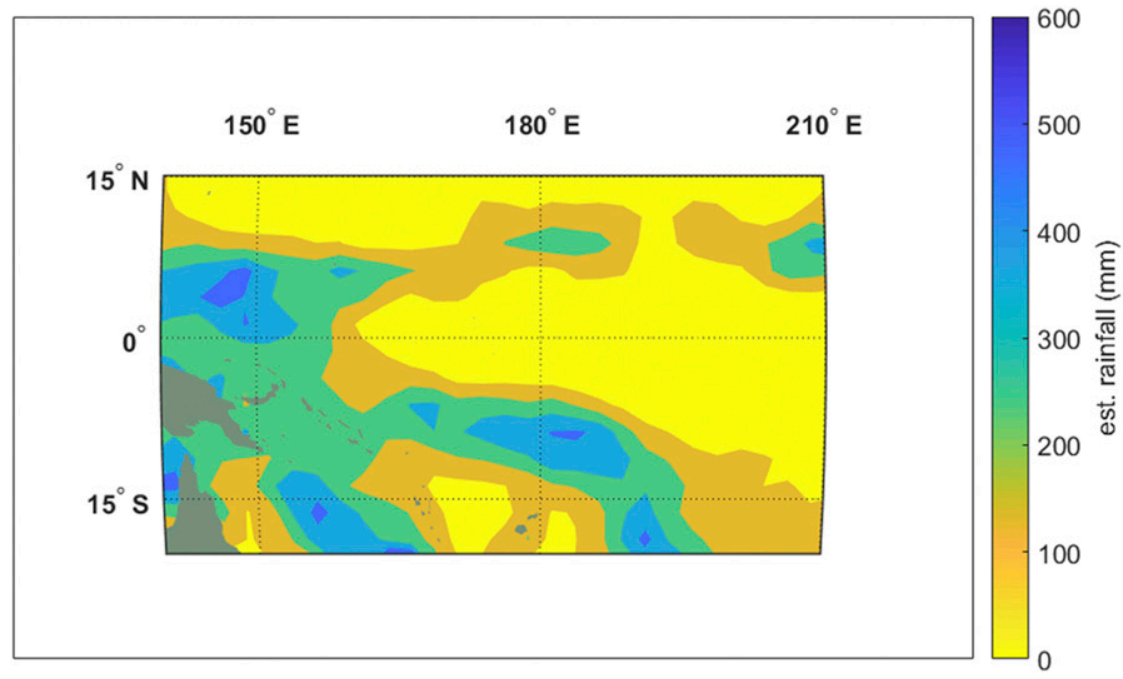

FIG. 14. GPCP rainfall estimates for January 1989 (La Niña).

The sparseness of this data presents fundamental challenges with respect to estimation and validation of rainfall and rainfall standard errors, including, but not limited to the following:

- An incomplete description of correlation structure in space-some lags are sampled much less than others

- Nonstationarity - the sparser the data, the more likely differences due to real spatial trends will exceed a particular threshold within groups of stations used for estimating semivariance or rainfall, resulting in degraded estimates

- Unsampled major rainfall features

- Regions of relatively large standard errors due to distances separating data points and the estimate location

- Large geographic gaps in cross-validation estimates
Determining the relative contributions of data sparseness and interpolation method, to the observed uncertainties of gridded and cross-validation estimates, is hampered by the lack of geographical regions in which observations are objectively dense, for example, one in which station separations are on the order of $1 \mathrm{~km}$ in all directions.

Ordinary kriging was chosen for its unbiasedness, its ability to produce theoretical standard error estimates, and its robustness relative to higher-order versions of kriging. The selection of ordinary kriging results in reduced estimate variance relative to the data used to generate the estimates, but in selecting estimation neighborhood size $(n=9)$ it was shown that back transformation of the square root-transformed rainfall estimates did not result in overall bias.

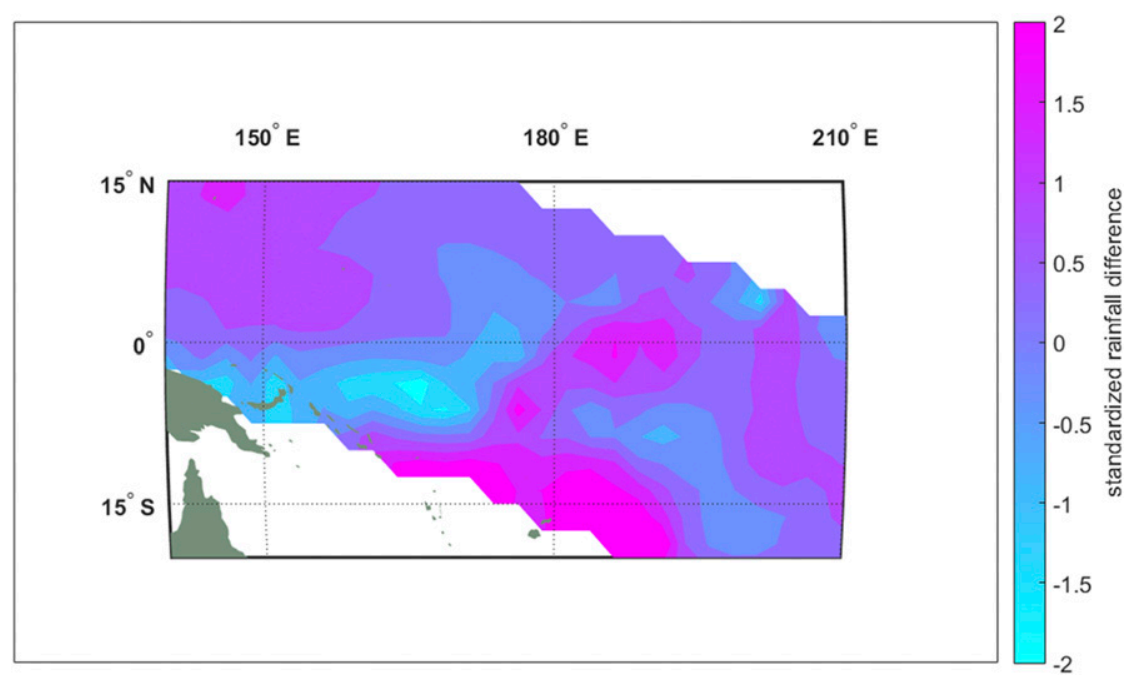

FIG. 15. Standardized rainfall difference January 1983 (El Niño) (kriging - GPCP)/kriging std error. 
The estimation method was cross validated by excluding observations from the semivariogram fitting procedure as well as the rainfall estimation procedure and then comparing those observations to estimates made for the same coordinates. Regressions of observed and estimated rainfall exhibit slopes that deviate from 1 by less than $8 \%$ and less than $6 \%$ for all months except August. Theoretical standard error estimates were shown to be highly predictive of observed standard errors, lending confidence in the standard errors accompanying the gridded product.

Gridded estimates of monthly rainfall and associated standard errors were generated and plots of rainfall for exemplar months were discussed and briefly compared to the current (version 2.3) monthly product published by the GPCP. The monthly rainfall estimates, along with transformed and back-transformed standard error estimates are published on the PACRAIN website in netCDF format. As of this writing, grids have been generated for the period of January 1930 to the most recent month available and are updated on a monthly basis, with typically a 2 - to 3 -month lag. The geographic coverage of the gridded product extends $15^{\circ}$ north and south of a line defined by lat $=60.85-0.3495$ lon where lon is given in positive degrees east of the prime meridian, and from $131^{\circ} \mathrm{E}$ eastward to $130^{\circ} \mathrm{W}$.

Additional updates to the gridded products will occur in the future, to possibly include incorporation of rain gauge data collected at nonatoll locations, anisotropic and/or local semivariograms. Given appropriate data coverage, semivariograms may also be generated uniquely for each month. Each of these updates are expected to result in improved geographic coverage of standard errors below a specified threshold. In the case of adding nonatoll data, the general coverage would also be increased. Standard errors at locations currently subject to gross extrapolation due to their distance from available atoll data, but rich with nonatoll data-such as broad swath between Papua New Guinea and Fiji-will benefit most.

Acknowledgments. This study was supported by NOAA's Global Ocean Monitoring and Observing Program. Cooperative Institute Contract NA160AR432115.

\section{REFERENCES}

Adler, R. F., and Coauthors, 2003: The version 2 Global Precipitation Climatology Project (GPCP) monthly precipitation analysis (1979present). J. Hydrometeor., 4, 1147-1167, https://doi.org/10.1175/ 1525-7541(2003)004<1147:TVGPCP > 2.0.CO;2.

, and Coauthors, 2018: The Global Precipitation Climatology Project (GPCP) monthly analysis (new version 2.3) and a review of 2017 global precipitation. Atmosphere, 9, 138, https://doi.org/10.3390/ atmos 9040138 .
Aldworth, J., and N. Cressie, 2003: Prediction of nonlinear spatial functionals. J. Stat. Plann. Inference, 112, 3-41, https://doi.org/ 10.1016/S0378-3758(02)00321-X.

Barnes, R., and T. Johnson, 1984: Positive kriging. Geostatistics for Natural Resources Characterization, G. Verly, Ed., Springer, 231-244.

Chiles, J. P., and P. Delfiner, 1999: Modeling Spatial Uncertainty. Wiley, 695 pp.

Cressie, N., 1985: Fitting variogram models by weighted least squares. Math. Geol., 17, 563-586, https://doi.org/10.1007/ BF01032109.

_ 1993a: Aggregation in geostatistical problems, Geostatistics Tróia '92, Vol. 1, Kluwer Academic, 25-36.

— 1993b: Statistics for Spatial Data. Wiley, 900 pp. , and C. Wikle, 2011: Statistics for Spatio-Temporal Data. Wiley, $588 \mathrm{pp}$.

Goovaerts, P., 2000: Geostatistical approaches for incorporating elevation into the spatial interpolation of rainfall. J. Hydrol., 228, 113-129, https://doi.org/10.1016/S0022-1694(00)00144-X.

Greene, J. S., M. Klatt, M. Morrissey, and S. Postawko, 2008: The Comprehensive Pacific Rainfall Database. J. Atmos. Oceanic Technol., 25, 71-82, https://doi.org/10.1175/2007JTECHA904.1.

Isaaks, E. H., and R. M. Srivastava, 1989: An Introduction to Applied Geostatistics. Oxford University Press, $561 \mathrm{pp}$.

Jewell, S., and N. Gaussiat, 2015: An assessment of kriging-based rain-gauge-radar merging techniques. Quart. J. Roy. Meteor. Soc., 141, 2300-2313, https://doi.org/10.1002/qj.2522.

Kastelec, D., and K. Kosmelj, 2002: Spatial interpolation of mean yearly precipitation using universal kriging. Developments in Statistics, A. Mrvar and A. Ferligoj, Eds., University of Ljubljana, 149-162.

Krajewski, W. F., 1987: Cokriging radar-rainfall and rain gage data. J. Geophys. Res., 92, 9571-9580, https://doi.org/10.1029/ JD092iD08p09571.

Lavoie, R. L., 1963: Some aspects of the meteorology of the tropical Pacific viewed from an atoll. Atoll Research Bulletin, No. 96, Pacific Science Board, Washington, DC, 80 pp.

Ly, S., C. Charles, and A. Degre, 2011: Geostatistical interpolation of daily rainfall at catchment scale: The use of several variogram models in the Ourthe and Ambleve catchments, Belgium. Hydrol. Earth Syst. Sci., 15, 2259-2274, https://doi.org/10.5194/ hess-15-2259-2011.

Matheron, G., 1963: Principles of geostatistics. Econ. Geol., 58 1246-1266, https://doi.org/10.2113/gsecongeo.58.8.1246.

Morrissey, M., 1991: Using sparse raingages to test satellite-based rainfall algorithms. J. Geophys. Res., 96, 18 561-18 571, https:// doi.org/10.1029/91JD01790.

— _ and J. Greene, 1993: Comparison of two satellite-based rainfall algorithms using Pacific Atoll raingage data. J. Appl. Meteor., 32, 411-425, https://doi.org/10.1175/1520-0450(1993) 032<0411:COTSBR $>2.0 . \mathrm{CO} ; 2$.

_ J. Maliekal, J. Greene, and J. Wang, 1995: The uncertainty of simple spatial averages using rain gauge networks. Water Resour. Res., 31, 2011-2017, https://doi.org/10.1029/95WR01232.

Park, N., P. Kyriakidis, and S. Hong, 2017: Geostatistical integration of coarse resolution satellite precipitation products and rain gauge data to map precipitation and fine spatial resolutions. Remote Sens., 9, 255-274, https://doi.org/10.3390/ rs9030255.

Postawko, S., M. Morrissey, and B. Gibson, 1994: The Schools of the Pacific Rainfall Climate Experiment: Combining research and education. Bull. Amer. Meteor. Soc., 75, 1260-1266, https://doi.org/10.1175/1520-0477-75.7.1249. 
Schuurmans, J., M. Bierkins, and E. Pebesbma, 2007: Automatic prediction of high-resolution daily rainfall fields for multiple extents: The potential of operational radar. J. Hydrometeor., $\mathbf{8}$, 1204-1224, https://doi.org/10.1175/2007JHM792.1.

Sideris, I., M. Gabella, R. Erdin, and U. Germann, 2014: Real-time radar-rain-gauge merging using spatio-temporal co-kriging with external drift in the alpine terrain of Switzerland. Quart. J. Roy. Meteor. Soc., 140, 1097-1111, https://doi.org/10.1002/qj.2188.

Szidarovszky, F., E. Baafi, and Y. Kim, 1987: Kriging without negative weights. Math. Geol., 19, 549-559, https://doi.org/ 10.1007/BF00896920.

Tang, Y., 2002: Comparison of semivariogram models for kriging monthly rainfall in eastern China. J. Zhejiang Univ. Sci., 3, 584-590, https://doi.org/10.1631/jzus.2002.0584.
Taylor, R. C., 1973: An atlas of Pacific Islands rainfall. University of Hawai'i at Mānoa Department of Meteorology Data Rep. $25,174 \mathrm{pp}$.

Verdin, A., B. Rajagopalan, W. Kleiber, and C. Funk, 2015: A Bayesian kriging approach for blending satellite and ground precipitation observations. Water Resour. Res., 51, 908-921, https://doi.org/ 10.1002/2014WR015963.

Wackernagel, H., 2003: Multivariate Geostatistics. Springer, 388 pp. Yamamoto, J., 2000: An alternative measure of the reliability of ordinary kriging estimates. Math. Geol., 32, 489-509, https://doi.org/10.1023/ A:1007577916868.

, 2005: Correcting the smoothing effects of ordinary kriging. Math. Geol., 37, 69-94, https://doi.org/10.1007/s11004-005-8748-7. 